\title{
GAIN AND DARK CURRENT STUDIES ON PLANAR PHOTODETECTORS MADE ON ANNEALED GaAs-ON-Si*
}

\author{
F. Riesz, Vo Van TUYen
}

Research Institute for Technical Physics of the Hungarian Academy of Sciences P.O. Box 76, 1325 Budapest, Hungary

\author{
AND J. VARRIO ${ }^{\dagger}$ \\ Department of Physics, Tampere University of Technology \\ P.O. Box 692, 33101 Tampere, Finland
}

Interdigital, planar photodetectors were fabricated from annealed GaAs/Si heterostructures grown by molecular beam epitaxy using alloyed $\mathrm{AuGe} / \mathrm{Ni}$ and non-alloyed $\mathrm{Cr} / \mathrm{Au}$ contacts. The dark current and optical gain of the $\mathrm{Cr} / \mathrm{Au}$ devices is higher than that of the $\mathrm{AuGe} / \mathrm{Ni}$ devices. Contact degradation due to annealing and a p-type background doping consistently explains our data. The gain-optical power relationship follows a power law with an exponent close to -1 .

PACS numbers: 73.50.Pz, 73.40.Sx, 85.60.Gz

The growth of GaAs on Si allows the integration of GaAs devices with the $\mathrm{Si}$ technology [1-2]. However, the $4.1 \%$ large lattice mismatch results in a high defect density in the epilayer which alters the physical properties of the epilayer and the devices made of it. In this work, we study the dark current and photoresponse properties of planar, interdigitated GaAs/Si photodetectors grown by molecular beam epitaxy (MBE). We analyze the effects of contact type, and compare our devices to those fabricated on Cr-doped, semiinsulating (SI) GaAs [3].

The Si substrate was (100) $p^{+}$-type $4^{\circ}$ miscut towards [011]. A nominally undoped $2 \mu \mathrm{m}$ layer was first grown by MBE using a two-step method. We applied an unusually high growth rate to enhance growth planarity, and consequently, an epilayer quality [2]: the $50 \mathrm{~nm}$ buffer layer was grown with a $5 \mu \mathrm{m} / \mathrm{h}$ growth rate at

\footnotetext{
*This work was supported, in part, by the (Hungarian) National Scientific Research Fund (OTKA) through grants F 4138 and F 16278 (in Budapest) and by the Technology Development Centre, Academy of Finland, Nokia Corporation, Wallac Ltd, and DCA Instruments Ltd. (in Tampere).

†Present address: Riihimäen Teknillinen Oppilaitos, Kaartokatu 2A, 11100 Riihimäki, Finland.
} 
$300^{\circ} \mathrm{C}$ followed by the final layer grown by $2 \mu \mathrm{m} / \mathrm{h}$ at $580^{\circ} \mathrm{C}$. The relatively streaky reflection high energy electron diffraction (RHEED) patterns during buffer growth indicated the enhanced planarity. The sample was then subjected to rapid thermal annealing (RTA) with a Si proximity cap at $930^{\circ} \mathrm{C} / 20 \mathrm{~s}$ to reduce the density of threading dislocations [4]. The $400 \mathrm{X}$-ray rocking curve halfwidth decreased from $390^{\prime \prime}$ to $210^{\prime \prime}$, indicating the beneficial effect of RTA.

A mesa etch was performed first down to the substrate to isolate the devices. Interdigital metal patterns were then formed by lift-off [5]. Devices with non-alloyed $\mathrm{Cr} / \mathrm{Au}(60 / 400 \mathrm{~nm})$ and alloyed AuGe/Ni/Au $(75 / 12 / 200 \mathrm{~nm})$ contacts were fabricated. Alloying was performed in forming gas at $350^{\circ} \mathrm{C}$, prior to $\mathrm{Cr} / \mathrm{Au}$ evaporation. Our contact technology was optimized to yield Schottky and ohmic contacts to $n$-type homoepitaxial GaAs, respectively; the actual contact behavior may be different in our case. The devices have $2 \mu \mathrm{m}$ nominal finger widths and finger spacings of 7 and $9.5 \mu \mathrm{m}$ for the ohmic, and 3.5 and $5 \mu \mathrm{m}$ for the Schottky devices. The diameter of the photosensitive area is about $100 \mu \mathrm{m}$.

The intrinsic dc optical gain $\mathrm{G}$ was inferred from the $I-V$ curves measured in the dark and at different illumination levels:

$$
\left.G=\left[\left(I-I_{\mathrm{d}}\right) / q\right] /\left[k P_{\mathrm{opt}} / h \nu\right)\right],
$$

where $I$ is the total current, $I_{\mathrm{d}}$ is the dark current, $P_{\mathrm{opt}}$ is the total incident optical power; $k$ is a correction factor for finger shadowing and surface reflectivity ( $k=0.44 \ldots 0.58$ depending on finger spacing). A 785 -nm-wavelength laser diode was used for illumination.
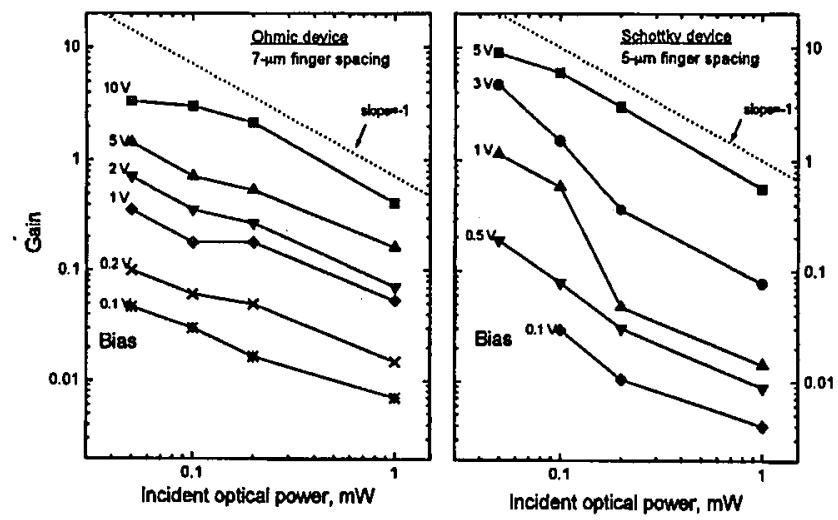

Fig. 1. The photoconductive gain as a function of bias and total incident optical power.

Typical $G\left(P_{\mathrm{opt}}\right)$ curves are shown in Fig. 1 with the bias voltage as a parameter. The gain of the Schottky devices is larger than that of the ohmic ones at the same bias, optical power and similar geometry, in contrary to devices with the same geometry and metallization but fabricated on Cr-doped, SI GaAs [3]. In those devices, the Schottky devices possess no gain but the ohmic devices do. This difference may have two origins: (i) It has been shown that the contact resistance of ohmic contacts to $\mathrm{GaAs} / \mathrm{Si}$ is much larger if RTA has been applied to 
GaAs [6]. This inhibits photoconductive gain but reduces dark current; (ii) The expected non-ideality of the Schottky contacts on the dislocated GaAs makes the $\mathrm{Cr} / \mathrm{Au}$ contact more injecting, causing better responsiveness but also a higher dark current as we found (Fig. 2). These features are probably enhanced by the slight $p$-type residual doping in the GaAs caused by Si diffusion from the substrate and the proximity-cap RTA process as proven by the SIMS and CV analysis on similar GaAs/Si samples [7].

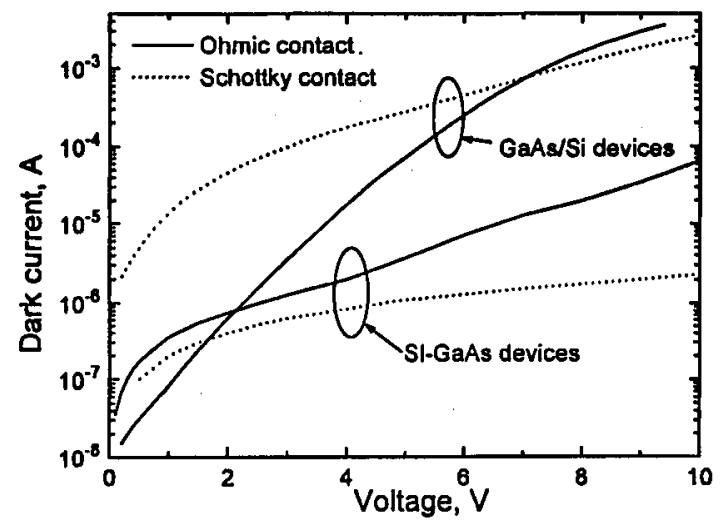

Fig. 2. The dark current of the GaAs/Si and the SI GaAs devices.

The $G\left(P_{\text {opt }}\right)$ relationship follows a power law with an exponent close to -1 in both type of devices (Fig. 1). This exponent is characteristic to a gain mechanism that is due to light-induced modulation of electrical barriers [8]. Such barriers may be present at the heterointerface, at the free surface, or around the threading dislocations or other defects.

The high dark currents are probably dominated by the current flowing through the large-area bonding pads and the conducting substrate, as supported by their insensitivity to the finger spacing. This dark current, although orders of magnitude higher than that of our SI GaAs detectors [3], is comparable to or better than that of other $\mathrm{GaAs} / \mathrm{Si}$ devices having similar geometries [9].

In conclusion, we have studied the gain and dark current of MBE-grown GaAs/Si planar photodetectors with different metallizations. Contact degradation and a $p$-type background doping consistently explains the differences in the dark current and optical gain of the devices having different contacts.

The authors are grateful to M. Németh-Sallay and her co-workers for device fabrication and $\mathrm{K}$. Wilke for illuminating discussions.

\section{References}

[1] P. Demeester, A. Ackaert, G. Coudenys, I. Moerman, L. Buydens, I. Pollentier, P. Van Daele, Prog. Cryst. Growth Charact. Mater. 22, 53 (1991).

[2] J. Varrio, Ph.D. Thesis, Tampere University of Technology, Tampere 1990. 
[3] F. Riesz, M. Serényi, M. Németh-Sallay, B. Szentpáli, Phys. Status Solidi A 143, K53 (1994).

[4] J. Varrio, F. Riesz, J. Lammasniemi, M. Hovinen, M. Pessa, Mater. Lett. 10, 49 (1990).

[5] F. Riesz, M. Németh-Sallay, J. Varrio, in: Proc. 8th Int. School on Cond. Matter Phys., 18-23 Sept. 1994, Varna, (Bulgaria), in press.

[6] K. Wilke, B. Budnick, M.H. Ludwig, G. Heymann, J. Appl. Phys. 77, 653 (1995).

[7] K. Wilke, G. Heymann, J. Varrio, M. Pessa, Inst. Phys. Conf. Ser. 129, 181 (1993).

[8] G.J. Papaioannou, J. Appl. Phys. 72, 5296 (1992); F. Riesz, Appl. Phys. A 60, 299 (1995).

[9] N.A. Papanicolaou, G.W. Anderson, J.A. Modolo, A. Georgakilas, Superlattices Microstruct. 8, 273 (1990). 